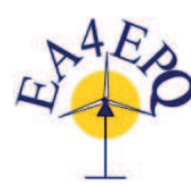

International Conference on Renewable Energies and Power Quality (ICREPQ'15)

La Coruña (Spain), $25^{\text {th }}$ to $27^{\text {th }}$ March, 2015

Renewable Energy and Pourer Qualily. Fournal (RE\&PQJ)

ISSN 2172-038 X, No.13, April 2015

\title{
A fault detection method for pitch actuators faults in wind turbines
}

\author{
C. Tutivén, Y. Vidal, L. Acho and J. Rodellar \\ Universitat Politècnica de Catalunya, \\ Applied Mathematics III Department, CoDAlab, \\ Comte d'Urgell, 187, 08036, Barcelona, Spain.
}

Phone number:+0034934137309, e-mail: christian.tutiven@upc.edu, yolanda.vidal@upc.edu leonardo.acho@upc.edu, jose.rodellar@upc.edu

\begin{abstract}
The paper presents a model-based fault detection method for pitch actuators faults using the normalized gradient method to estimate the parameters of the pitch actuator. One major difficulty is that the input signal to the parametric estimation method must be a persistent excitation. To circumvent this, a chattering term is added to the pitch control law and the usual low-pass filters are not used for the parametrization in the normalized gradient method (thus acceleration information is used). In order to verify the proposed method, simulations are conducted within a Hardware in the Loop (HiL) platform using the wind turbine simulation software FAST (Fatigue, Aerodynamics, Structures, and Turbulence).
\end{abstract}

\section{Key words}

Parameter estimation, fault detection, wind turbine, FAST, Hardware in the Loop.

\section{Introduction}

Wind energy is rapidly emerging as a cost-effective sustainable technology. The demand of higher power production installation and the continuous increase of the size of wind turbines (WT) have led to new challenges in the WT systems. Fault detection and isolation (FDI) techniques are fundamental to detect and locate degradations and failures in the operation of WT components as early as possible. In consequence to this, the FDI research has witnessed a steady increase in interest in this application area. Publications can be named, among others, such as an $\mathrm{H}$-infinity based technique to detect and estimate the magnitude of blade bending moment and pitch actuators faults [1], an unknown input observer designed for the detection of sensor faults around the wind turbine drive train [2], a model-based system identification technique for pitch actuator faults [3], FDI and fault tolerant control (FTC) for a wind turbine generator and converter system [4] and doubly fed induction generator sensor fault diagnosis [5]. The FDI and FTC systems use fault detection (FD) techniques to achieve their goals. A FD technique detects faults by means of a residual signal produced by available measurements. It must be a signal that is close to zero in the absence of a fault, and significantly affected in the presence of faults [6], [7], [8]. The main components of a fault detection system are the following [6], [7]: a residual generator signal, residual evaluation method, and a prescribed threshold to decide whether a fault occurs or not [6], [7], [9].

This paper proposes a model-based fault detection method that detects a fault whenever a change occurs in the dynamics of the pitch actuator [10]. The normalized gradient method [11] is used to estimate the parameters of the pitch actuator and a residual signal is obtained. A 5MW wind turbine based on FAST will be used in the simulations. Moreover, tests will be conducted in a HiL platform using the Arduino microcontroller [17].

The paper is organized as follows. In Section 2, the National Renewable Energy Laboratory (NREL) wind turbine simulator FAST [12] code, the pitch actuator model and the generator and converter model are given. Section 3 states the control strategies. The FD method is conceived in Section 4. Finally, in Section 5 the Hardware in the Loop platform, and the simulations are presented.

\section{Wind Turbine Model}

\section{A. FAST}

The FAST code [12] is an aerolastic simulator capable of predicting the extreme and fatigue loads of two and three bladed Horizontal Axis Wind Turbines (HAWTs). This simulator was developed by the National Renewable Energy Laboratory (NREL) and has been accepted by the scientific community and is used by many researchers in the development of new control systems for wind turbines. We select this simulator for validation due to the fact that in 2005 the Germanischer Lloyd WindEnergie evaluated FAST and found it suitable for the calculation of onshore wind turbine loads for design and certification [13]. Numerical validation with FAST were performed with the NREL 5MW on-shore wind turbine. The wind turbine characteristics are summarized in Table I, see [16]. 


\begin{tabular}{lc}
\hline Rated power & $5 \mathrm{MW}$ \\
Number of blades & 3 \\
Height of tower & $87.6 \mathrm{~m}$ \\
Rotor diameter & $126 \mathrm{~m}$ \\
Cut in, Rated, Cut-Out Wind Speed & $3 \mathrm{~m} / \mathrm{s}, 11.4 \mathrm{~m} / \mathrm{s}, 25 \mathrm{~m} / \mathrm{s}$ \\
Gearbox ratio & 98 \\
Nominal generator speed $\left(\Omega_{n g}\right)$ & $1173.7 \mathrm{rpm}$ \\
\hline
\end{tabular}

TABLE I

WT Characteristics.

New wind data sets are generated with TurbSim [14], a stochastic, full-field, turbulent-wind simulator developed by NREL. This wind inflow (see Fig. 1) is used in the simulations of Section 5. Note that the mean wind speed at the hub height is $18.5 \mathrm{~m} / \mathrm{s}$, thus the wind turbine is working in the full load region (also called Region 3).

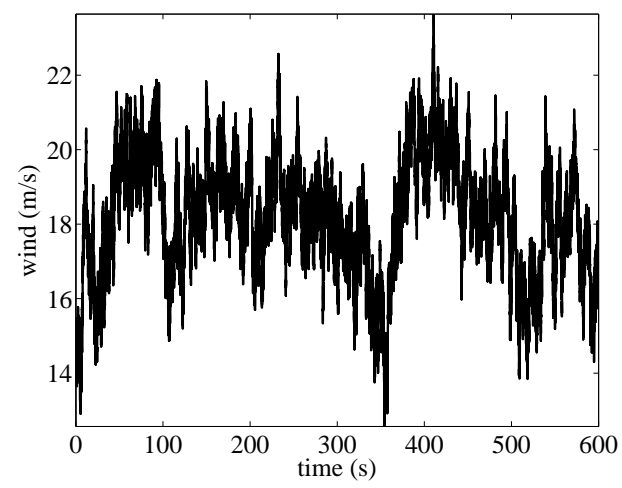

Fig. 1. Wind speed profile.

\section{B. Pitch actuator model}

The hydraulic pitch system can be modeled by a second order system, see [10], with reference angle $\beta_{r_{i}}$ and actual pitch angle $\beta_{i}$ as

$$
\frac{\beta_{i}(s)}{\beta_{r_{i}}(s)}=\frac{\omega_{n}^{2}}{s^{2}+2 \zeta \omega_{n} s+\omega_{n}^{2}}, \quad i=1,2,3,
$$

where $\zeta$ is the damping factor and $\omega_{n}$ is the natural frequency. This transfer function is associated to the control system of the pitch angle of every blade $(i=1,2,3)$ and the values of its parameters are identical when no fault exists. For the healthy case, the parameters $\zeta=0.6$ and $\omega_{n}=11.11 \mathrm{rad} / \mathrm{s}$ are used [10]. When a fault occurs, these parameters will change.

The proposed FD method will estimate the values of $\zeta$ and $\omega_{n}$ and, whenever a change in these estimated values is observed, a fault can be detected.

\section{Generator and converter model}

The generator and converter dynamics are modeled by a first-order transfer function [15]

$$
\frac{\tau_{g}(s)}{\tau_{g, r}(s)}=\frac{\alpha_{g c}}{s+\alpha_{g c}}
$$

where $\tau_{g}$ is the generator torque, $\tau_{g, r}$ is the torque reference to the generator and $\alpha_{g c}$ is the generator and converter model parameter (in the simulations $\alpha_{g c}=50$ [16]). The electrical power produced by the generator is given by

$$
P_{g}(t)=\eta_{g} \Omega_{g}(t) \tau_{g}(t),
$$

where $\eta_{g}$ is the generator efficiency (in the simulations $\eta=$ $0.98)$ and $\Omega_{g}$ is the generator speed measurement. See Fig. 2.

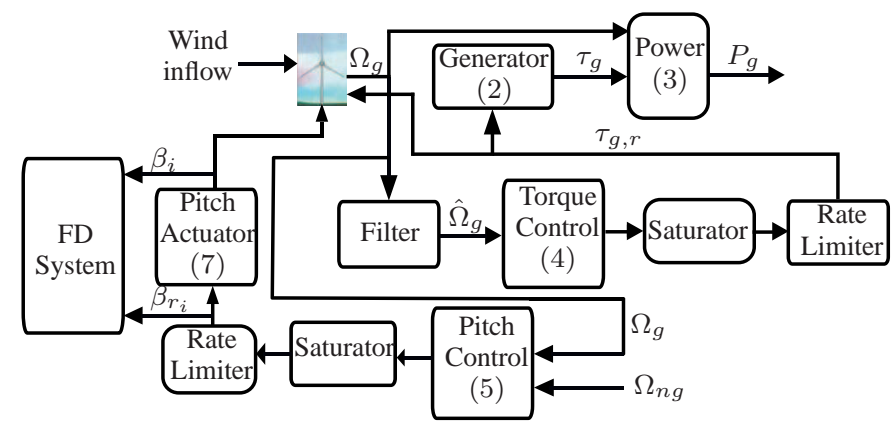

Fig. 2. Block diagram. Note that the torque control is allocated in a controller hardware (see, Section 5).

\section{Control Strategy}

\section{A. Torque control}

The 5MW reference wind turbine given by FAST contains a torque controller. In Region 3, this controller maintains regulated the generated power, thus the generator torque is proposed inversely proportional to the filtered generator speed [12], or,

$$
\tau_{g}=\frac{P_{r}}{\hat{\Omega}_{g}},
$$

where $P_{r}$ is the electrical rated power, and $\hat{\Omega}_{g}$ is the filtered generator speed. Note that $\hat{\Omega}_{g}$ is used as proposed by [16] to mitigate high-frequency excitation by using a recursive, single-pole low-pass filter with exponential smoothing.

As the generator may not be able to supply the desired electromechanic torque depending on the operating conditions, and in the case of overshooting, the torque controller is saturated to a maximum of $47402.9 \mathrm{Nm}$ and a maximum rate limit of $15000 \mathrm{Nm} / \mathrm{s}$ [16]. See Fig. 2.

This controller will be allocated in a controller hardware (see [17]) performing a HiL simulation.

\section{B. Pitch control}

To assist the torque control with regulating the wind turbine electrical power output, while avoiding significant loads and maintaining the rotor speed within acceptable limits, a pitch control is added to the generator speed tracking error. The pitch angle control is a gain scheduling PI-controller [16] to which we add a chattering term, that is 
where

$\beta_{r_{i}}=K_{p}(\gamma)\left(\Omega_{g}-\Omega_{n g}\right)+K_{i}(\gamma) \int_{0}^{t}\left(\Omega_{g}-\Omega_{n g}\right) d \tau+K_{\alpha} \operatorname{sgn}\left(\Omega_{g}-\Omega_{n g}\right)$,

$$
i=1,2,3
$$

where $\Omega_{n g}$ is the nominal generator speed (at which the rated electrical power of wind turbine is obtained) and the scheduling parameter $\gamma$ is obtained by averaging the measurements of all pitch angles as

$$
\gamma=\frac{\sum_{i=1}^{3} \beta_{i}}{3}
$$

The scheduled gains are calculated following [16]. In the numerical simulation, $K_{\alpha}$ is set to 0.05 . Finally, to not exceed the mechanical limitations of the pitch actuator, the input signal $\beta_{r_{i}}$ is saturated to a maximum of $45^{\circ}$ and a rate limit of $8^{\circ}$ s [16]. See Fig. 2. Actually, this rate limit acts as a low-pass filter. This is one of the main difficulties for the parameter estimation method as this is the input signal which should be a persistent excitation.

Note that to obtain a greater excitaton signal, Eq. (5) uses an unfiltered generator speed $\left(\Omega_{g}\right)$ and an added chattering term. Moreover, the unfiltered generator speed improves the regulation of the generated power as has been observed in numerical simulations.

\section{Fault Detection Method}

This section describes the proposed method to estimate the pitch actuator parameters given in (1) as well as the design of two residual signals for FD purposes.

Equation (1) can be written as

$$
\frac{Y(s)}{U(s)}=\frac{\omega_{n}^{2}}{s^{2}+2 \zeta \omega_{n} s+\omega_{n}^{2}},
$$

where $Y(s)$ is the actual angle $\beta_{i}(s)$, and $U(s)$ is the reference angle $\beta_{r_{i}}(s)$. In time domain

$$
\omega_{n}^{2} u=\ddot{y}+2 \zeta \omega_{n} \dot{y}+\omega_{n}^{2} y,
$$

and isolating the acceleration

$$
\ddot{y}=\omega_{n}^{2} u-2 \zeta \omega_{n} \dot{y}-\omega_{n}^{2} y,
$$

that can be rewritten as

$$
\ddot{y}=\left(\omega_{n}^{2},-2 \zeta \omega_{n},-\omega_{n}^{2}\right) \quad\left(\begin{array}{l}
u \\
\dot{y} \\
y
\end{array}\right)=\theta^{T} \phi,
$$

where $\theta^{T}$ is the vector of parameters and $\phi$ is the regression vector.

Using the normalized gradient method [11], we obtain the following estimation

$$
\hat{\ddot{y}}=\left(\hat{\theta}_{1}, \hat{\theta}_{2}, \hat{\theta}_{3}\right)\left(\begin{array}{l}
u \\
\dot{y} \\
y
\end{array}\right)=\hat{\theta}^{T} \phi,
$$

$$
\dot{\hat{\theta}}=\frac{-\rho e \phi}{1+k \phi^{T} \phi} ; \quad k>0,
$$

where $\rho$ is a positive number called the estimator gain, $k$ is a design parameter (for the simulation $\rho=20$ and $k=100$ ) and $e$ is the error defined by

$$
e=\hat{\ddot{y}}-\ddot{y} .
$$

Note that $\hat{\theta}_{1}, \hat{\theta}_{2}$ and $\hat{\theta}_{3}$ are the estimations of $\omega_{n}^{2},-2 \zeta \omega_{n}$ and $-\omega_{n}^{2}$, respectively, and $\ddot{y}$ is the acceleration signal.

Remark: Note that the usual low-pass filters are not utilized in the parametrization and therefore acceleration information is employed. Different parameter estimation methods were tested with and without the usual low-pass filters and the results showed that the estimated values approached much faster the real values when the filters were not used.

We propose the following residual signals $r_{1}$ and $r_{2}$

$$
\begin{gathered}
r_{1}=\hat{\ddot{y}}-\ddot{y}_{h}, \\
r_{2}=\left\|\hat{\theta}-\theta_{h}\right\|,
\end{gathered}
$$

where $\theta_{h}=\left(\omega_{n}^{2},-2 \zeta \omega_{n},-\omega_{n}^{2}\right)^{T}=(123,-13,-123)^{T}$ are the healthy parameter values and $\ddot{y}_{h}$ is the healthy pitch actuator acceleration which is numerically obtained using

$$
\ddot{y}_{h}=\omega_{n}^{2} u-2 \zeta \omega_{n} \dot{y}_{h}-\omega_{n}^{2} y_{h},
$$

with the healthy parameter values.

\section{Experimental Setup}

\section{A. Hardware in the Loop}

In this section, the experimental setup used for the simulations is explained. The dynamics of the wind turbine are simulated in FAST, which emulates all the input signals needed by the controllers. The torque controller is implemented in the open source Arduino microcontroller, which will be connected via USB to a computer where the turbine is simulated. Fig. 3 shows the experimental configuration of the HiL, for more details see [17].

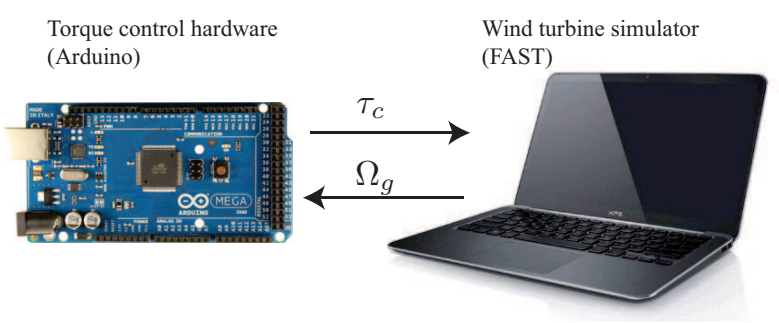

Fig. 3. Diagram of the experimental configuration of HiL.

The proposed HiL platform allows to test the performance of the torque controller when running in real-time and a fault 
exists in the pitch actuator. Note that testing these cases experimentally can seriously damage the WT, thus a HiL approach is preferable.

\section{B. Fault description}

The considered fault is a high air content in the oil [10]. Air is much more compressible than oil, so it changes the dynamics of the hydraulic actuator. The fault is modeled by changing the parameters $\zeta$ and $\omega_{n}$ in the pitch actuator model of blade 3 (the other blades are healthy). The used values for this faulty case are $\zeta=0.9$ and $\omega_{n}=3.42$ (see [10]). The fault is linearly introduced from 300 s to 320 s and is fully active from 320 s to 600 s (see [10]).

\section{Simulation results}

To finally setup the FD method a threshold is prescribed for the residual signals using their maximum values (in absolute value) in the healthy case. For $r_{1}$ the prescribed threshold is 0.4 and for $r_{2}$ is 0.85 . Fig. 4 shows that with signal $r_{1}$ the fault is detected at around 308s, while Fig. 5 shows that with signal $r_{2}$ the fault is detected at around 302s. In both cases the fault is detected before it is fully active, i.e. during its linear introduction.

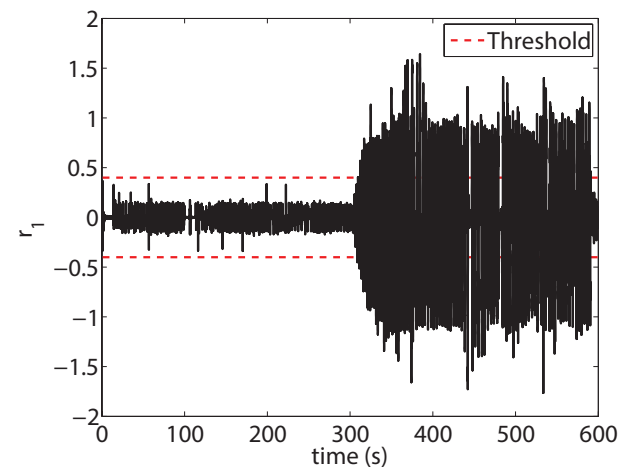

Fig. 4. Residual signal $r_{1}$.

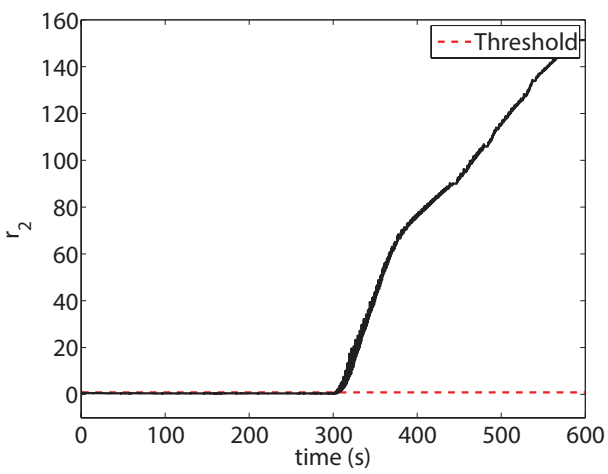

Fig. 5. Residual signal $r_{2}$.

In Fig. 6, 7 and 8 it can be seen that, when a fault appears, the estimated parameters $\hat{\theta}_{1}, \hat{\theta}_{2}$ and $\hat{\theta}_{3}$, in a very short time, differ from the healthy reference values as they start the (slow) convergence to the faulty reference values. Recall that the main contribution of this work is a FD method and this is accomplished even with a slow parameter convergence.

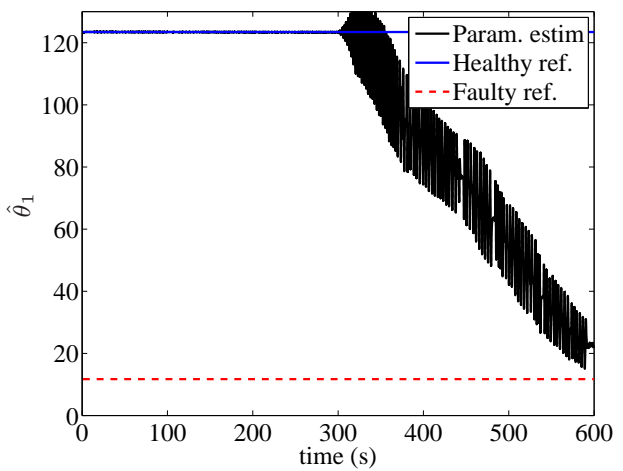

Fig. 6. Estimation of $\theta_{1}$.

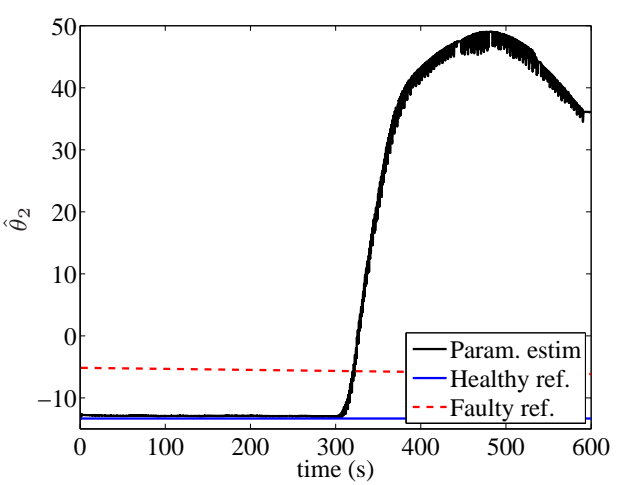

Fig. 7. Estimation of $\theta_{2}$.

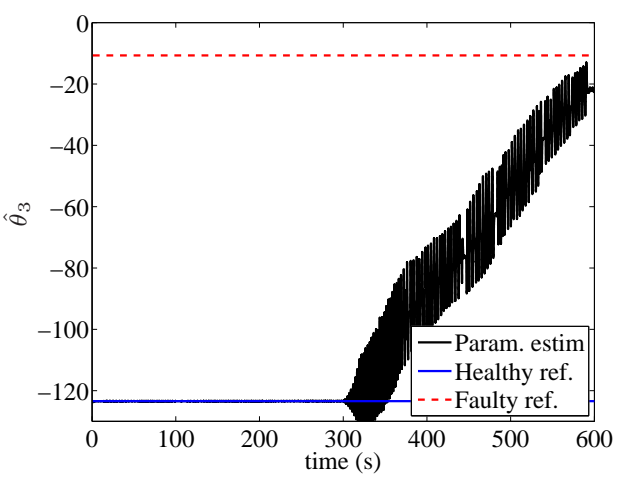

Fig. 8. Estimation of $\theta_{3}$.

The following results will show that the proposed closedloop is robust against the studied fault.

Fig. 9 shows that the torque controller has an acceptable performance, maintaining the generated power closed to the desired value (5 MW) despite the appearance of a fault at time 300s. From Fig. 10, the torque action of the proposed controller achieves reasonable values. 


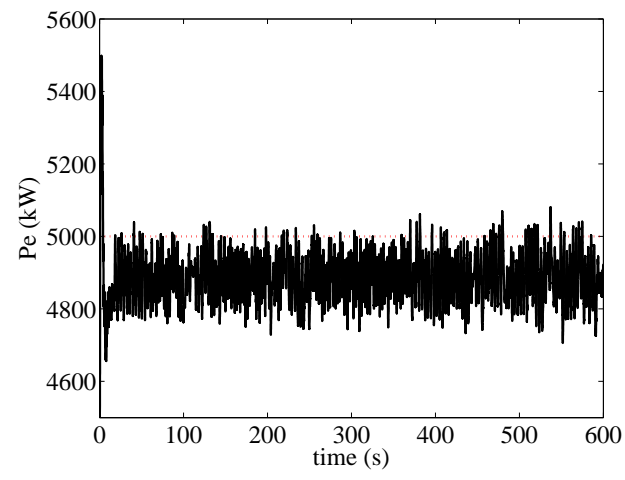

Fig. 9. Generated electrical power.

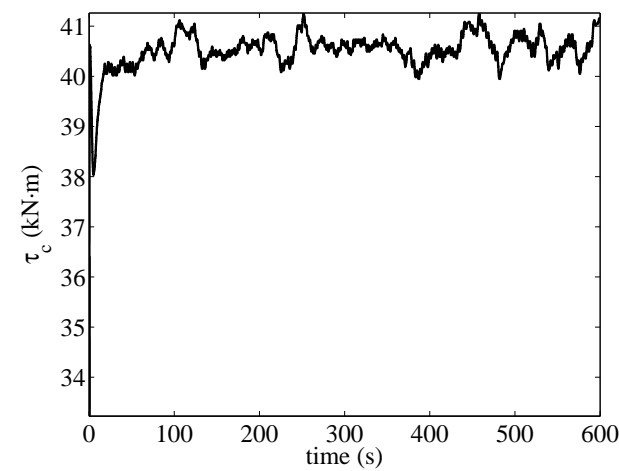

Fig. 10. Torque control.

The generator speed, as shown in Fig. 11 is near its nominal value due to the controllers action. Figures 12 and 13 show that the pitch control signal is regulated within the authorized variation domain. That is none of the variations exceed the mechanical limitations of the pitch actuator. Note also that, when all the pitch actuators are in healthy condition all blades have the same behavior, however when a fault exists in one of them a difference between the variation of the pitch angle 1 (healthy) and the pitch angle 3 (faulty) can be observed.

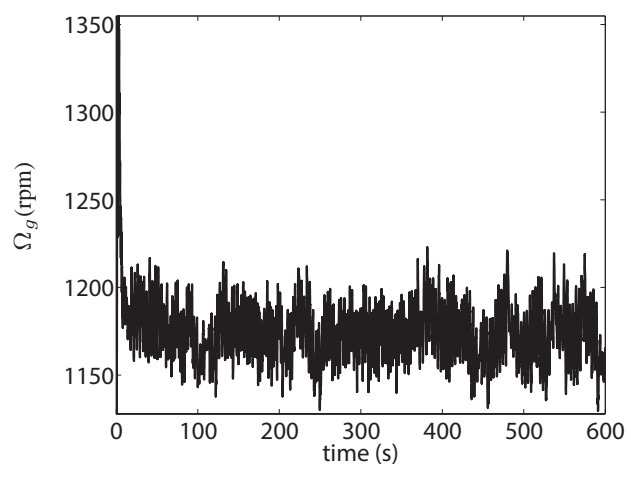

Fig. 11. Generator speed.

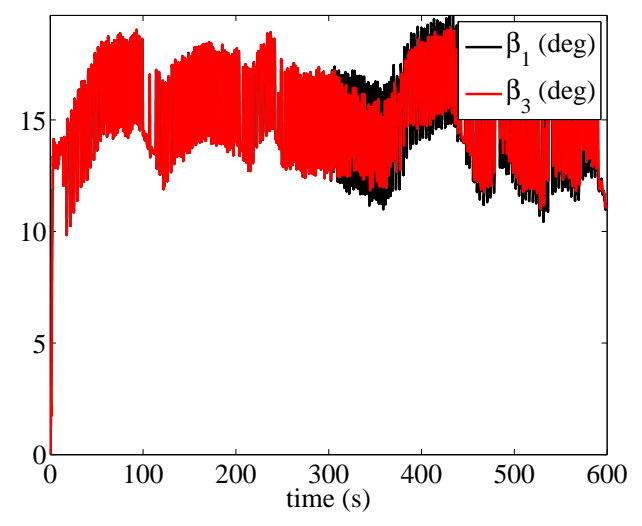

Fig. 12. Pitch control.

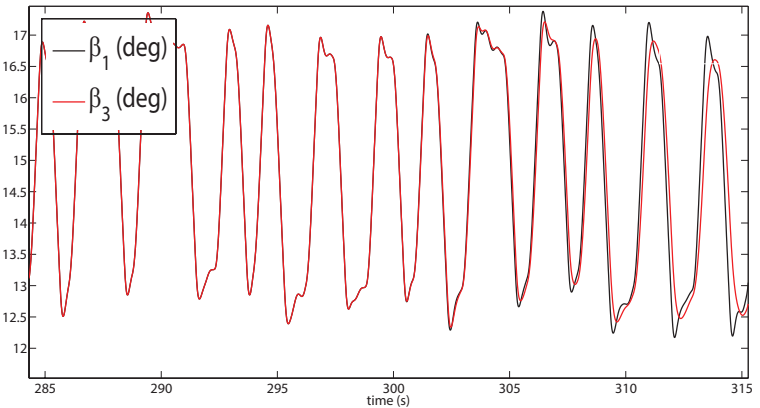

Fig. 13. Zoom of the pitch control signal.

It can be seen in Figures 14, 15, 16 and 17 that the fore-aft and the side-to-side accelerations at different tower heights are of the same magnitude than the case presented in [16] (where a gain-scheduling PI is used for the pitch controller). Thus the addition of the chattering term helps to the FD method without much variation in the tower accelerations.

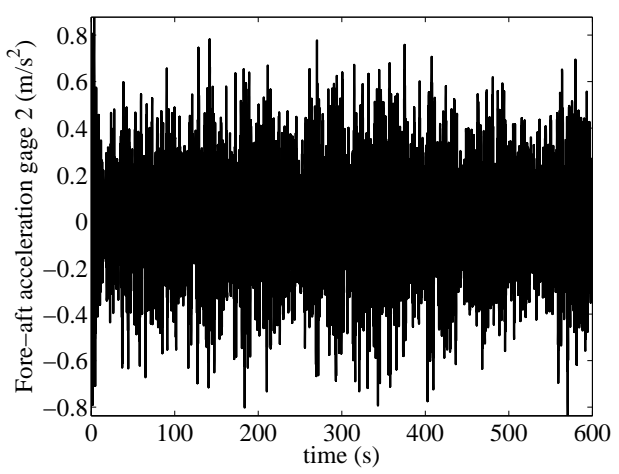

Fig. 14. Fore-aft accelerations at mid-tower. 


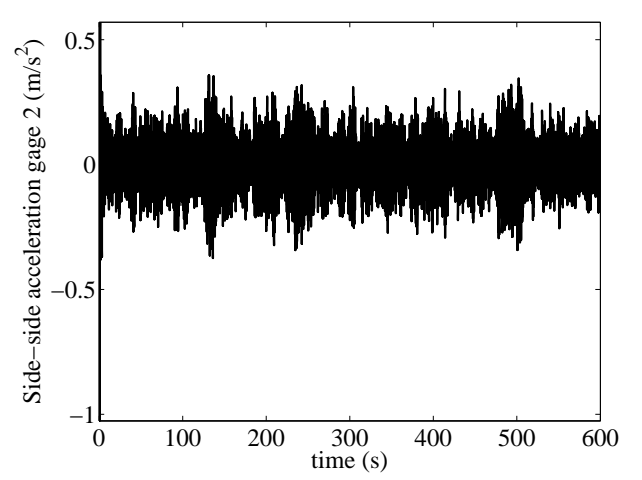

Fig. 15. Side-to-side acceleration at mid-tower.

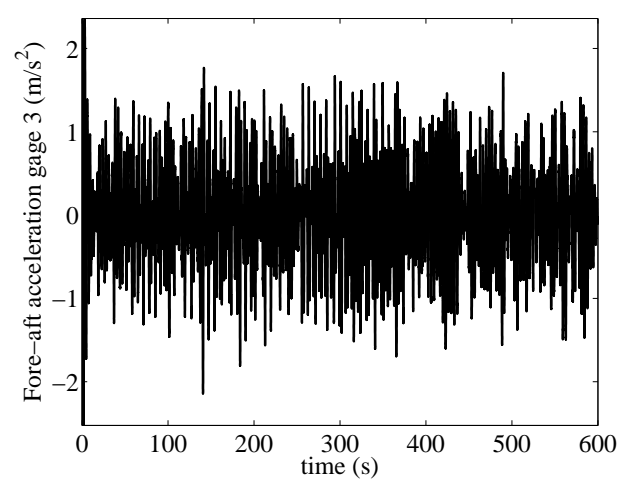

Fig. 16. Fore-aft acceleration at tower top.

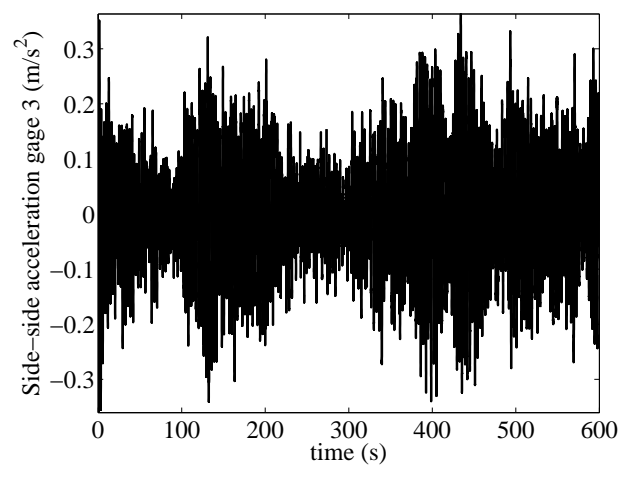

Fig. 17. Side-to-side acceleration at tower top.

\section{Conclusions}

A WT fault detection method for pitch actuator faults is studied in this paper. Although only one type of fault is presented in the simulations, any fault that implies a change in the dynamics of the pitch actuator will be detected using this method. Note that, even if the approximation of the estimated parameters to the fault values is slow, the two proposed residual signals detect in short notice the appearance of the fault. Moreover, according to the experiments, the overall closed-loop system is robust against the studied faults. The numerical simulations in HiL platform have demonstrated the performance of the proposed fault detection method.

\section{References}

[1] Wei, X.; Verhaegen, M.; van den Engelsen, T. Sensor fault detection and isolation for wind turbines based on subspace identification and kalman filter techiniques. International Journal of Adaptive Control and Signal Processing 24:687-707, 2010.

[2] Odgaard, P.F.; Stoustrup, J.; Nielsen, R.; Damgaard, C. Observer based detection of sensor faults in wind turbines. In Proceedings of European Wind Energy Conference. 2009.

[3] Donders, S. Fault detection and identification for wind turbine systems: a closed-loop analysis. University of Twente (Masters thesis). 2002.

[4] Odgaard, P.F.; Stoustrup, J. Unknown input observer based scheme for detecting faults in a wind turbine converter. In Proceedings of 7th IFAC Symposium on Fault Detection, Supervision and Safety of Technical Processes. 2009.

[5] Galvez, M.R. Sensor fault diagnosis for wind driven doubly fed induction generators. Universite Libre de Bruxelles (Phd. thesis) 2010.

[6] Vidal, Y.; Acho, L.; Pozo, F. Robust fault detection in hysteretic base isolation systems. Mechanical Systems and Signal Processing 29:447456, 2012.

[7] Liberatore, S.; Speyer, J.L.; Hsu, A.C. Application of a fault detection filter to structural health monitoring. Automatica 42:1199-1209, 2006.

[8] Besançon, G. High gain observation with disturbances attenuation and application to robust fault detection. Automatica 39:1095-1102,2003.

[9] Edwards, C.; Spurgeon S.K.; Patton, R.J. Sliding mode observers for fault detection and isolation. Automatica 36:541-553. 2000.

[10] Odgaard, F.; Johnson, K. Wind turbine fault detection and fault tolerant control - an enhanced benchmark challenge. American Control Conference (ACC) 4447-4452. 2013.

[11] Tao, G.; Adaptive control design and analysis. Wiley Interscience. 2003.

[12] Jonkman, J.M.; Buhl, M.L. FAST user's guide. http://www.wind.nrel.gov/designcodes/simulators/fast/. 2005

[13] Manjock, A. Design codes FAST and ADAMS for load calculations of onshore wind turbines. National Renewable Energy Laboratory (NREL). 2005.

[14] Kelley, N.; Jonkman, B. NWTC computer-aided engineering tools (Turbsim).

URL http://wind.nrel.gov/designcodes/preprocessors/turbsim/. 2013.

[15] Odgaard, P.F.; Stoustrup, J.; Kinnaert, M. Fault-tolerant control of wind turbines: a benchmark model. Transactions on Control Systems Technology. 21:1168-1182. 2013.

[16] Jonkman, J.; Buttereld, S.; Musial, W.; Scott, G. Definition of a 5 $M W$ reference wind turbine for offshore system development. National Renewable Energy Laboratory. 2009.

[17] Vidal, Y.; Acho, L.; Luo, N; Tutivén, C. Hardware in the loop wind turbine simulator for control system testing. pp.449-466 in N, Luo; Y, Vidal and L, Acho (Eds.), Wind turbine control and monitoring. Springer. 2014. 